\title{
Metalloproteinase-2 expression correlates with aggressiveness of cutaneous squamous cell carcinomas
}

\author{
Olga Fundyler ${ }^{1,2}$, Manish Khanna ${ }^{3}$ and Bruce R Smoller ${ }^{3,4}$ \\ ${ }^{1}$ Department of Pathology; ${ }^{2}$ Department of Dermatology, Beth Israel Deaconess Medical Center, Boston, MA, \\ USA; ${ }^{3}$ Department of Dermatology and ${ }^{4}$ Department of Pathology, University of Arkansas for Medical \\ Sciences, Little Rock, AR, USA
}

\begin{abstract}
Matrix metalloproteinases compose a family of enzymes involved in degradation of the extracellular matrix. Tumor cells must penetrate the basement membrane and traverse the extracellular matrix in order to invade surrounding structures and metastasize to distant sites. Gelatinases, particularly gelatinase A (matrix metalloproteinase-2), demonstrate degradative activity against components of the basement membrane and may be involved in the progression of in situ squamous cell carcinoma lesions. Matrix metalloproteinase-2 overexpression has been correlated with tumor invasiveness and metastasis in a wide variety of cancer types, including squamous cell carcinoma arising on mucous membranes. However, correlation between matrix metalloproteinase-2 overexpression and the spread and prognosis of cutaneous squamous cell carcinoma has not been characterized in the literature at present. In this study, we used immunohistochemical techniques to examine the expression of matrix metalloproteinase- 2 in 10 actinic keratosis, 15 in situ, 13 invasive, 13 primary (with documented metastatic disease), 11 metastatic, and 8 recurrent squamous cell carcinoma cases. We found that while the average staining intensity (scale $0-3+, 3+$ being strongest) of actinic keratosis and in situ lesions was not statistically significant (0.87-1.3 and $0.75-1.4$, respectively), the average staining intensity of invasive squamous cell carcinomas (1.6-2.5) was significantly greater than that of actinic keratosis and in situ lesions. Likewise, while the average staining intensity of primary squamous cell carcinomas and metastatic squamous cell carcinomas was not found to be statistically significant (3.1-3.5 and 3.1-3.8, respectively), the average staining intensity of these lesions was significantly higher than that of invasive lesions. We also found that the intensity of matrix metalloproteinase-2 staining correlates with cellular atypia, inflammation, neovascularization, and the invasive tumor front, as well as tumor aggressiveness, and may play a role in the pathogenesis, invasion, and metastasis of cutaneous squamous cell carcinoma.

Modern Pathology (2004) 17, 496-502, advance online publication, 27 February 2004; doi:10.1038/modpathol.3800066
\end{abstract}

Keywords: matrix metalloproteinase; gelatinase; cutaneous squamous cell carcinoma; invasion; metastasis; immunohistochemistry

Matrix metalloproteinases compose a family of enzymes involved in the degradation of extracellular matrix. Matrix metalloproteinase activity is directed against essentially all elements of the extracellular matrix. Thus far, 21 matrix metalloproteinases have been identified in human tissue and are categorized according to substrate specificity. Subclasses include collagenases, gelatinases, matrilysins, strome-

Correspondence: Dr BR Smoller, Department of Pathology, University of Arkansas for Medical Sciences, 4301 W. Markham St., Little Rock, AR 72205, USA.

E-mail: Smollerbrucer@uams.edu

Received 9 September 2003; revised 13 December 2003; accepted 15 December 2003; published online 27 February 2004 lysins, membrane-type matrix metalloproteinases, and several others. ${ }^{-3}$

Tumor cells must penetrate the basement membrane and traverse the extracellular matrix in order to invade surrounding structures and metastasize to distant sites. Gelatinases, particularly gelatinase A (matrix metalloproteinase-2), demonstrate degradative activity against components of the basement membrane and may be involved in the progression of in situ lesions. ${ }^{4,5}$ Matrix metalloproteinase-2 has affinity for gelatin; collagen I, IV, VII, X; tenascin; fibrillin; osteonectin; fibronectin; and monocyte chemoattractant protein $3 .{ }^{1}$ Collagenase IV activity is particularly associated with basement membrane compromise. ${ }^{4,6}$

Matrix metalloproteinase-2 overexpression has been correlated with tumor invasiveness and metas- 
tasis in a wide variety of cancer types, ${ }^{6-15}$ including squamous cell carcinoma arising on mucous membranes. ${ }^{16-19}$ A significant increase in matrix metalloproteinase-2 expression has been observed in invasive and metastatic oral squamous cell carcino$\mathrm{ma}^{6,17,19}$ and has been associated with a shorter disease-free survival., ${ }^{40}$ However, correlation between matrix metalloproteinase-2 overexpression and the spread and prognosis of cutaneous squamous cell carcinoma has not been characterized in the literature at present. The differing pathogeneses of squamous cell carcinoma arising from mucous and cutaneous sites suggests the possibility of discrete molecular means of invasiveness. For instance, hypermethylation inactivation of p16 is instrumental in many mucosal squamous cell carcinomas but does not appear to play a role in cutaneous squamous cell carcinoma. ${ }^{21}$

Available treatment of metastatic cutaneous squamous cell carcinoma with chemotherapy and radiation fails to offer a favorable prognosis. Several matrix metalloproteinase inhibitors are currently under investigation for therapeutic use, and many target a wide variety of matrix metalloproteinases including matrix metalloproteinase-2. Among these are peptidomimetic and nonpeptidic inhibitors, natural matrix metalloproteinase inhibitors, tetracycline derivatives, and bisphosphanates. ${ }^{1}$ The development of more specific matrix metalloproteinase inhibitors, such as matrix metalloproteinase inhibitor-166, may lead to a decrease in side effects. Matrix metalloproteinase inhibitor-166 inhibits matrix metalloproteinase-2, -9 , and $-14 .^{22,23}$

In this study, we compare matrix metalloproteinase-2 expression in cutaneous squamous cell carcinoma specimens representing precancerous (actinic keratosis), in situ, locally invasive, recurrent, and metastatic disease. Our goal is to examine for the presence of a correlation between the degree of matrix metalloproteinase-2 expression and tumor aggressiveness. If such correlation should exist, it is possible that matrix metalloproteinase- 2 could be used as a tumor marker for metastasis and that some subset of patients with cutaneous squamous cell carcinoma may benefit from matrix metalloproteinase inhibitor chemotherapeutic treatment.

\section{Materials and methods}

Immunohistochemistry was used to determine the expression of matrix metalloproteinase-2 in 10 actinic keratosis, 15 in situ, 13 invasive, 13 primary (with documented metastatic disease), 11 metastatic, and eight recurrent squamous cell carcinoma cases. Formalin-fixed, paraffin-embedded biopsy tissue was retrieved from archival material. Sections $(4 \mu \mathrm{m})$ were heated at $60^{\circ} \mathrm{C}$ for $24 \mathrm{~h}$ prior to deparaffinization in xylene and rehydration in ethanol. Antigen retrieval was achieved by incubation at $120^{\circ} \mathrm{C}$ in citrate buffer ( $\mathrm{pH} \mathrm{6.1)}$ for $20 \mathrm{~min}$ in a high-pressure oven. The sections were then washed in Tris-buffered saline (TBS) buffer ( $\mathrm{pH}$ 7.6) and stained using mouse antihuman matrix metalloproteinase-2 MAb (NeoMarkers matrix metalloproteinase-2 Ab-4 VC2) and goat biotinylated antimouse IgG Ab (Lab Vision Corporation, Fremont, USA; Ca HRP/AEC TQ-015-HA). Incubation with mouse antihuman MAb was carried out for $30 \mathrm{~min}$ at a 1:50 dilution in the TBS buffer. The sections were washed in the TBS buffer between each incubation. Hematoxylin counterstain was applied for $1 \mathrm{~min}$. Slides were then rinsed in distilled water and mounted.

Results were determined by three independent investigators. Staining intensity was determined subjectively using a $0-3$ scale. Tumor cells staining equivalent to that of basal keratinocytes in normal skin were assigned a rating of $1+$. Tumor cells staining less intensely than cells of the basal layer received a rating of 0 . Tumor cells staining as strongly as endothelial cells received a rating of $3+$. Staining intensity between 1 and 3 received a rating of $2+$. The basal layer of normal skin, when present, and endothelial cells were used as an internal control in each slide.

\section{Results}

We have examined expression of matrix metalloproteinase-2 in 10 actinic keratosis, 15 in situ, 13 invasive, 13 primary (with documented metastatic disease), 11 metastatic, and eight recurrent squamous cell carcinoma cases. Normal skin showed weak staining of the basal layer of the epidermis (Figure 2), as well as strong staining of endothelial cells (Figure 3). All biopsy specimens in this study also showed this pattern of staining. In addition, cells comprising the inflammatory infiltrate present in many of the slides stained $2+$ to $3+$ in intensity (Figure 4). Endothelial cells in areas consistent with neovascularization stained $3+$.

Nine $(90 \%)$ actinic keratosis lesions stained with $1+$ intensity $(n=10)$, and one stained with $2+$ $(10 \%)$ intensity. The $2+$ staining in the latter specimen corresponded with acantholysis. Other actinic keratosis specimens showed an epidermal blush corresponding with acanthosis, while the normal skin flanking the lesions lacked stain superficial to the basal cell layer (Figure 4). Staining occurred in a granular pattern and appeared strongest in the cytoplasm. The average staining intensity of actinic keratosis lesions was 1.1, with a range of 0.87-1.3 (significance level $=0.5$; see Figure 1 ).

In all, $12(80 \%)$ in situ lesions stained with $1+$ intensity $(n=15)$. One $(7 \%)$ in situ specimen was negative for stain, two (13\%) exhibited $2+$ staining, and one $(7 \%)$ showed a combined area of $2+$ and $3+$ staining. The in situ lesions containing $2+$ and $3+$ staining exhibited an immunohistochemical pattern revealing increased staining intensity correlating 


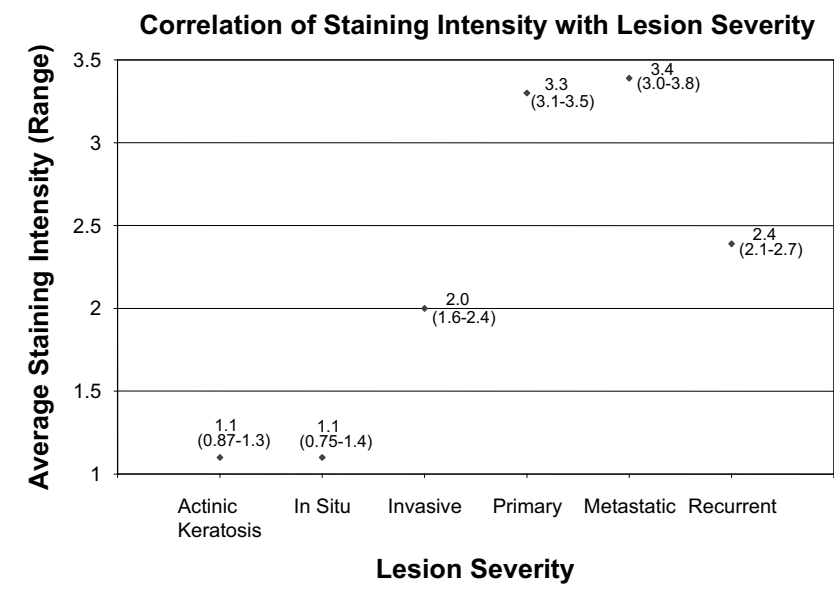

Figure 1 Correlation of staining intensity with lesion severity.

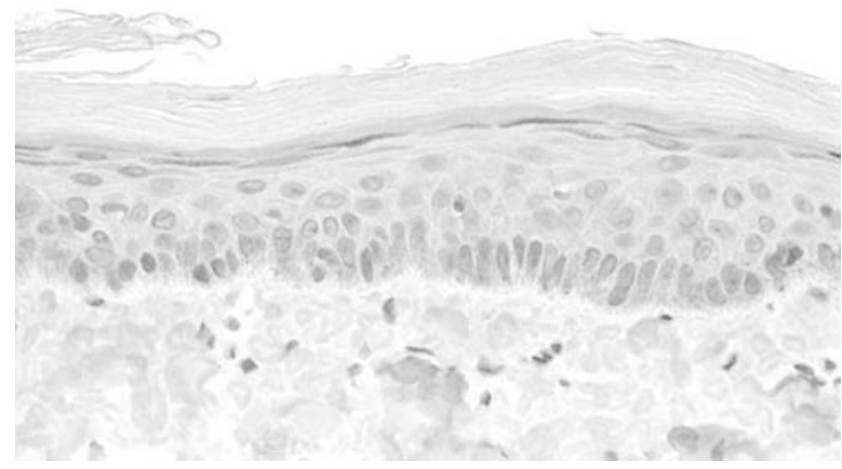

Figure 2 Normal skin. The matrix metalloproteinase-2 stain is greatly limited to the basal cell layer. This degree of staining represents $1+$ staining intensity.

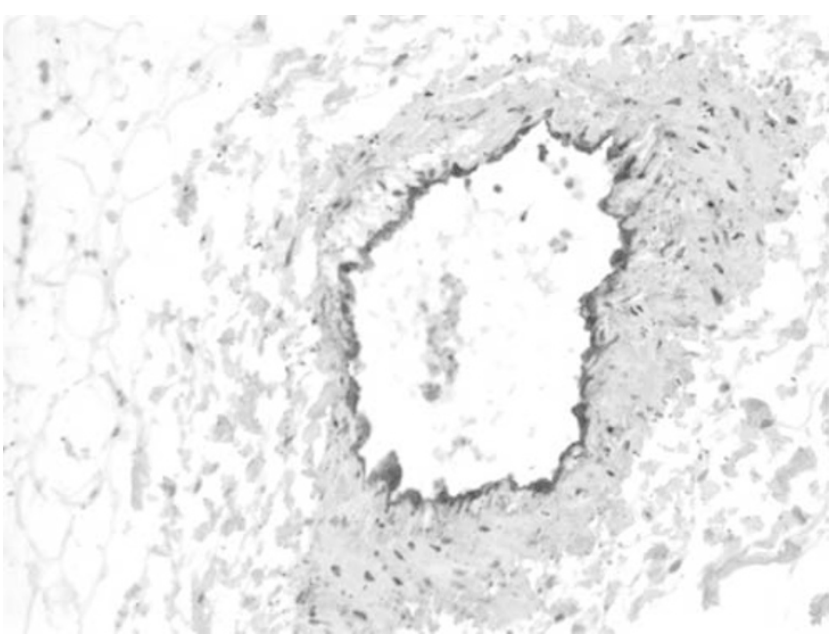

Figure 3 Normal vessel. Endothelial cells stain $3+$ in intensity.

with acantholysis. As with actinic keratoses, the strongest areas of stain were observed in the cytoplasm, where staining appeared granular in

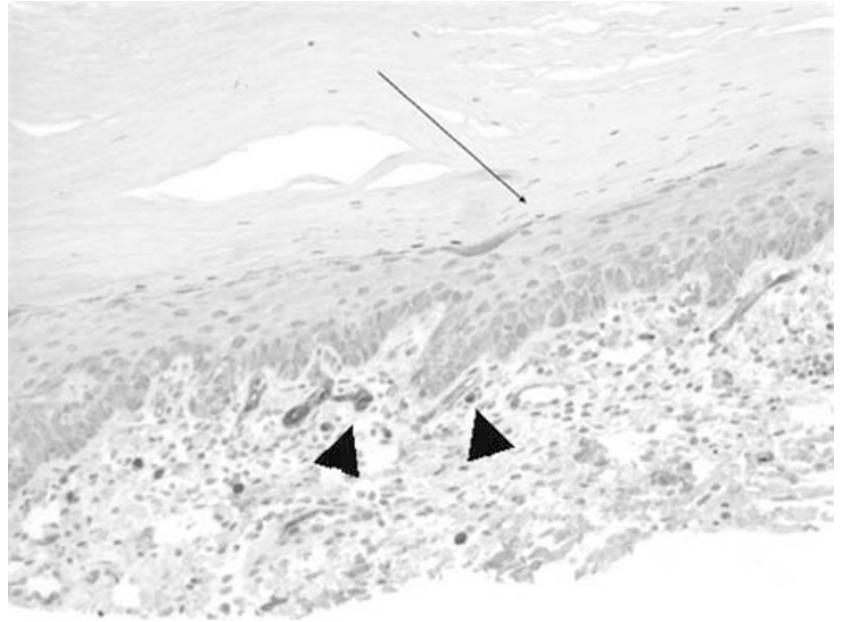

Figure 4 Transition between normal skin and actinic keratosis. The small arrow indicates the transition between normal skin and acanthosis. Note the correlation between increase in epidermal stain and degree of acanthosis. The large arrows point to inflammatory cells, which stain $3+$ in intensity.

nature. Figure 5 shows a representative in situ specimen in which matrix metalloproteinase-2 stain is distributed throughout the lesion. The average staining intensity of in situ lesions was 1.1, with a range of 0.75-1.4 (significance level $=0.5$; see Figure 1).

One $(8 \%)$ invasive squamous cell carcinoma specimen was negative for stain $(n=13)$. Three $(23 \%)$ invasive squamous cell carcinomas stained with $1+$ intensity, four (31\%) stained with $2+$ intensity, and $5(38 \%)$ exhibited $3+$ staining. Staining was granular in nature, cytoplasmic in distribution, and strongest at the invasive front. Stain was often distributed throughout the lesion (Figure 6). Acantholytic cells expressed greater staining intensity (Figure 7). The average staining intensity of invasive squamous cell carcinoma lesions was 2.0, with a range of 1.6-2.4 (significance level $=0.5$; see Figure 1 ).

One $(8 \%)$ primary squamous cell carcinoma, with documented metastatic disease $(n=13)$, exhibited $1+$ staining intensity, three $(23 \%)$ exhibited $2+$ staining, and nine (69\%) primary squamous cell carcinomas exhibited $3+$ staining intensity. Staining was often splotchy, with areas of acantholysis staining more strongly than their less architecturally disordered counterparts. Staining remained granular and cytoplasmic. The invasive fronts of these tumors often exhibited markedly increased staining intensity and were bordered by areas of inflammatory infiltrate and neovascularization, cells in both of which also stained intensely (Figure 8). The average staining intensity of primary squamous cell carcinoma lesions was 3.3, with a range of 3.1-3.5 (significance level $=0.5$; see Figure 1).

One $(9 \%)$ metastatic squamous cell carcinoma exhibited $1+$ staining intensity $(n=11)$, three $(27 \%)$ metastatic lesions exhibited $2+$ staining, and seven (64\%) exhibited $3+$ staining. As seen 


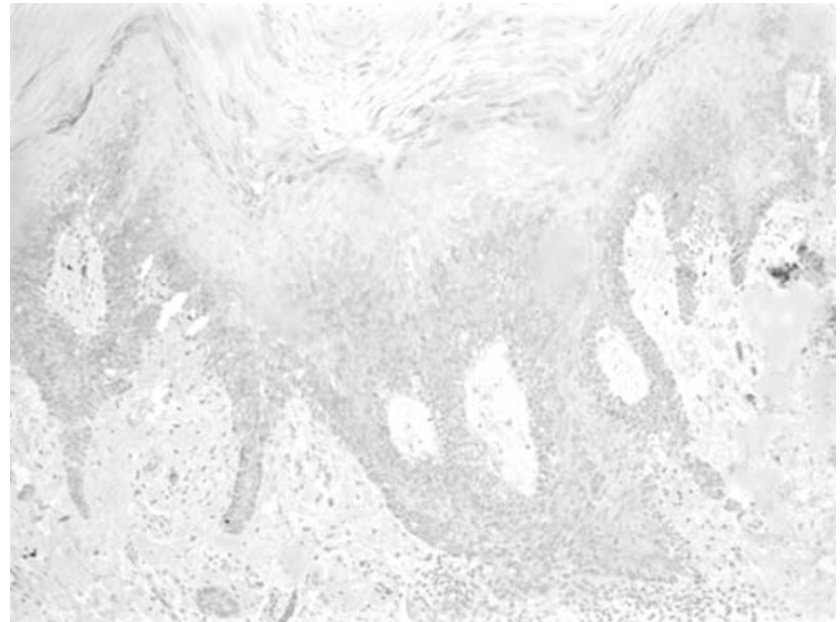

Figure 5 Squamous cell carcinoma in situ. Matrix metalloproteinase-2 staining is seen throughout the in situ lesion.

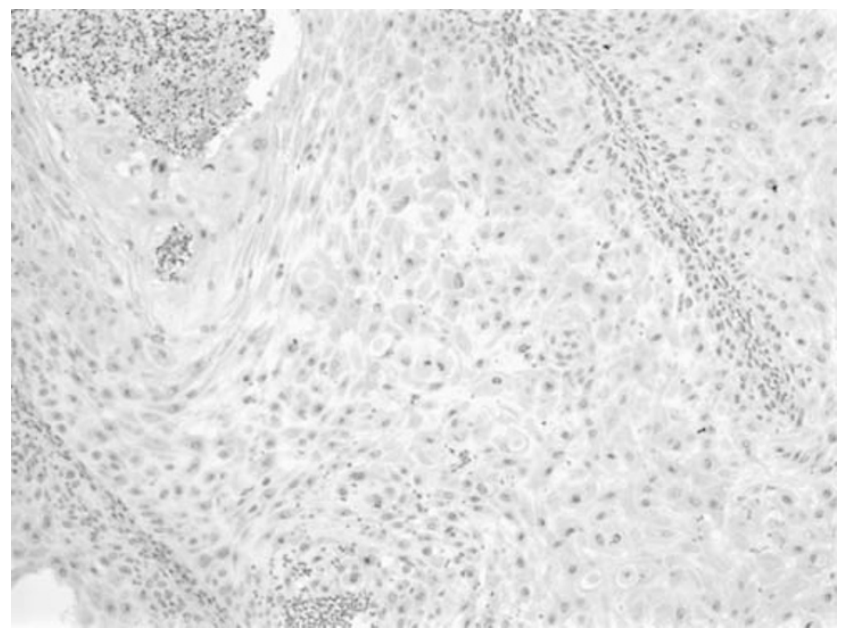

Figure 6 Invasive squamous cell carcinoma. Matrix metalloproteinase-2 staining is seen throughout the invasive squamous cell carcinoma lesion.

with primary lesions, the granular, cytoplasmic staining in metastatic cases was often splotchy, corresponding to the frequently encountered acantholytic cells. Increased staining was observed at the stromal interface. The average staining intensity of metastatic lesions was 3.4, with a range of 3.0-3.8 (significance level $=0.5$; see Figure 1).

One $(13 \%)$ recurrent squamous cell carcinoma exhibited $1+$ staining intensity $(n=8)$, three $(38 \%)$ recurrent squamous cell carcinomas exhibited $2+$ staining, and four $(50 \%)$ exhibited $3+$ staining. The immunohistochemical pattern observed in these lesions was very similar to that seen in primary lesions with associated metastases. Splotchy staining corresponded to areas of acantholysis. Staining was granular and cytoplasmic in location. The invasive front often exhibited increased staining intensity and was met with an intensely staining inflammatory infiltrate and areas of neovasculariza-

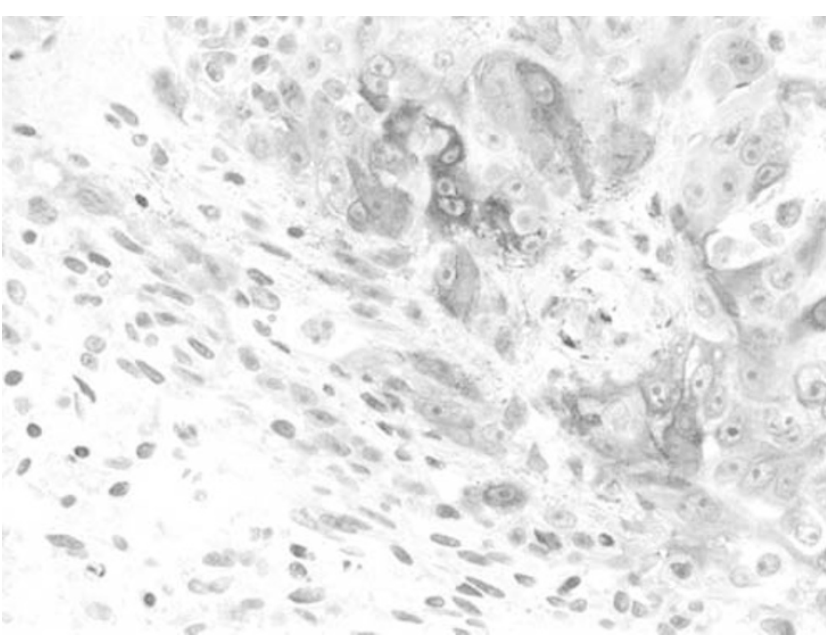

Figure 7 Acantholytic cells in an invasive squamous cell carcinoma. The acantholytic cells stain more intensely than their more normal-appearing counterparts.

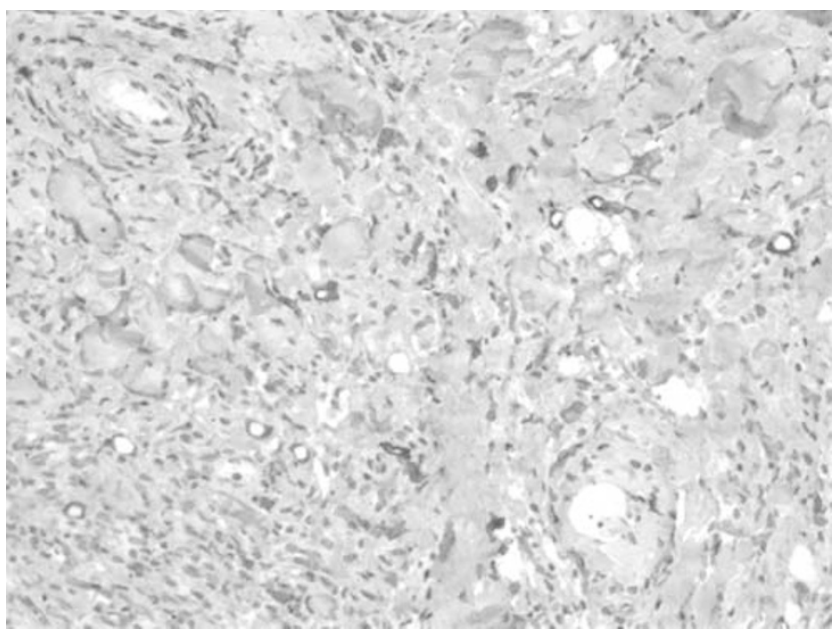

Figure 8 Invasive tumor front. Tumor cells at the invasive front exhibit $3+$ staining intensity and are met with an area of neovascularization. Endothelial cells also stain $3+$.

tion. The average staining intensity of recurrent lesions was 2.4, with a range of 2.1-2.7 (significance level $=0.5$; see Figure 1).

\section{Discussion}

Cellular atypia in squamous cell carcinoma has previously been correlated with tumor metastasis and decreased patient survival. ${ }^{24}$ In this study, we found that cellular atypia was also associated with increased matrix metalloproteinase-2 staining intensity. For instance, the majority of actinic keratosis and in situ specimens exhibited $1+$ staining. The few cases in which these lesions stained with greater intensity exhibited such staining only in acantholytic cells, presumably secondary to deranged formation of desmosomes. In fact, 
acantholytic cells also stained more intensely than surrounding, more normal-appearing tumor cells, in invasive, primary, metastatic, and recurrent lesions.

If cellular atypia, manifested, at least in part as acantholysis, is associated with both metastatic disease and increased matrix metalloproteinase-2 staining, then matrix metalloproteinase-2 may play a role in the progression of cutaneous squamous cell carcinoma lesions. Matrix metalloproteinase-2 has been associated with tumor progression, metastasis, and poor prognosis in various different cancers, including those of pancreatic, liver, lung, renal, bladder, gastric, ovarian, and mucosal squamous cell carcinoma origins. ${ }^{6-19}$ Matrix metalloproteinase2 appears to play a role in the degradation of extracellular matrix components, including type IV collagen. ${ }^{1-3}$ Compromise of the basement membrane, of which type IV collagen is a key component, is a vital step in invasion and metastasis. ${ }^{1,4}$ As tumor cells displaying atypia are associated with tumor progression, we assume that such cells possess characteristics, which promote basement membrane degradation. Since matrix metalloproteinase-2 has the capacity to digest type IV collagen enzymatically and is over expressed in the most atypical cells of cutaneous squamous cell carcinoma tumors and in situ specimens, we suspect that matrix metalloproteinase-2 is involved in the pathogenesis of these lesions. Inflammatory infiltration has also been correlated with tumor metastasis and poor prognosis. ${ }^{24}$ We observed that cells of the inflammatory infiltrate associated with the tumor mass in many of our squamous cell carcinoma specimens stained with $2-3+$ intensity and were often bordered by intensely staining malignant cells. This immunohistochemical pattern was most pronounced at the invasive front, an area of the tumor that is particularly active in invasion and metastasis. ${ }^{25-27}$ The correlation between intensely staining inflammatory infiltration and intensely staining tumor cells at the invasive front suggests an interaction, between inflammatory and tumor cells, which increases matrix metalloproteinase-2 expression and promotes tumor progression.

The literature supports an interaction between tumor cells and the inflammatory infiltrate that promotes invasion and metastasis. For instance, cells of the inflammatory infiltrate, including fibroblasts, associated with the tumor mass have been shown to secrete various growth factors and cytokines that increase the production of proteins such as matrix metalloproteinases in tumor cells. The reverse also occurs. Thus, inflammatory cells and fibroblasts activate, and in turn are activated by, surrounding tumor cells. The result is increased production of factors that participate in tumor virulence by promoting, for example, angiogenesis and degradation of the extracellular matrix. ${ }^{5,28}$ Our finding of increased matrix metalloproteinase-2 staining in tumor cells near the inflammatory infiltrate is consistent with the literature and suggests that matrix metalloproteinase-2 may interact with the inflammatory infiltrate to further cutaneous squamous cell carcinoma progression.

Neovascularization is a significant event in the process of metastasis of a variety of tumor types. ${ }^{24}$ We found that areas of neovascularization were often associated with intensely staining tumor cells, particularly at the invasive front. This pattern of staining was most pronounced in primary, metastatic, and recurrent squamous cell carcinoma specimens. Increased matrix metalloproteinase-2 staining near areas of vascular permeation in cases of advanced disease further supports a role for matrix metalloproteinase- 2 in the metastasis of cutaneous squamous cell carcinoma.

Increased staining intensity correlating with more advanced stages of cutaneous squamous cell carcinoma indicates that matrix metalloproteinase-2 may play a role in the pathogenesis and progression of these lesions. While the average staining intensity of actinic keratosis and in situ lesions was not statistically significant $(0.87-1.3$ and $0.75-1.4$, respectively; see Figure 1), the average staining intensity of invasive squamous cell carcinomas (1.6-2.5) was significantly greater than that of actinic keratosis and in situ lesions. Likewise, while the average staining intensity of primary squamous cell carcinomas and metastatic squamous cell carcinomas was not found to be statistically significant (3.1-3.5 and 3.1-3.8, respectively), the average staining intensity of these lesions was significantly higher than that of invasive lesions. The average staining intensity of recurrent squamous cell carcinomas (2.1-2.7) was significantly lower than that of primary and metastatic lesions, and the reason for this finding is uncertain.

Staining of the invasive group of squamous cell carcinomas in this study may be misleading. We found that 31 and $38 \%$ of these specimens, for which no metastatic disease was documented, stained $2+$ and $3+$, respectively. Such staining intensity, although still consistent with our hypothesis, is impressive for this group of lesions. However, we did not have follow-up beyond 1-2 years for most of these patients, and it is possible that some of these invasive cancers may indeed represent lesions with great metastatic potential. Future research endeavors will test this hypothesis.

Some authors have reported an association between various matrix metalloproteinases and histologic grade in a variety of tumors. ${ }^{29-32}$ We did not find a correlation between matrix metalloproteinase2 expression, as measured by immunohistochemical analysis, and the grade of cutaneous squamous cell carcinoma (grading data not shown).

In conclusion, we found that the degree of matrix metalloproteinase-2 expression, as documented by immunohistochemical analysis, correlates with cutaneous squamous cell carcinoma tumor aggressiveness. Additionally, as the intensity of matrix metalloproteinase-2 staining correlates with cellular 
atypia, inflammation, neovascularization, and the invasive tumor front, matrix metalloproteinase-2 may play a role in the pathogenesis, invasion, and metastasis of cutaneous squamous cell carcinoma. It is thus possible that in the future matrix metalloproteinase-2 may be used as a tumor marker for metastasis and that some subset of patients with cutaneous squamous cell carcinoma may benefit from matrix metalloproteinase inhibitor chemotherapeutic treatment targeted against matrix metalloproteinase-2.

\section{References}

1 Vihinen P, Kahari VM. Matrix metalloproteinases in cancer: prognostic markers and therapeutic targets. Int J Cancer 2002;99:157-166.

2 O-Charoenrat P, Rhys-Evans PH, Eccles SA. Expression of matrix metalloproteinases and their inhibitors correlates with invasion and metastasis in squamous cell carcinoma of the head and neck. Arch Otolaryngol Head Neck Surg 2001;127:813-820.

3 Curran S, Murray GI. Matrix metalloproteinases in tumour invasion and metastasis. J Pathol 1999;189: 300-308.

4 Yoshizaki T, Maruyama Y, Sato H, et al. Expression of tissue inhibitor of matrix metalloproteinase-2 correlates with activation of matrix metalloproteinase-2 and predicts poor prognosis in tongue squamous cell carcinoma. Int J Cancer 2001;95:44-50.

5 Kanekura T, Chen X, Kanzaki T. Basigin (CD147) is expressed on melanoma cells and induces tumor cell invasion by stimulating production of matrix metalloproteinases by fibroblasts. Int J Cancer 2002;99:520-528.

6 Monig SP, Baldus SE, Hennecken JK, et al. Expression of matrix metalloproteinase-2 is associated with progression and lymph node metastasis of gastric carcinoma. Histopathology 2001;39:597-602.

7 Davidson B, Goldberg I, Kopolovic J, et al. Matrix metalloproteinase-2 and TIMP-2 expression correlates with poor prognosis in cervical carcinoma-a clinicopathologic study using immunohistochemistry and mRNA in situ hybridization. Gynecol Oncol 1999;73: 372-382.

8 Hrabec E, Strek M, Nowak D, et al. Activity of type IV collagenases (matrix metalloproteinase-2 and matrix metalloproteinase-9) in primary pulmonary carcinomas: a quantitative analysis. J Cancer Res Clin Oncol 2002;128:197-204.

9 Krecicki T, Zalesska-Krecicka M, Jelen M, et al. Expression of type IV collagen and matrix metalloproteinase-2 (type IV collagenase) in relation to nodal status in laryngeal cancer. Clin Otolaryngol 2001;26: 469-472.

$10 \mathrm{Xu} \mathrm{K}$, Hou S, Du Z. Prognostic value of matrix metalloproteinase-2 and tissue inhibitor of metalloproteinase-2 in bladder carcinoma. Chin Med J 2002; 115:743-745.

11 Sakata K, Shigemasa K, Nagai N, et al. Expression of matrix metalloproteinases (matrix metalloproteinase-2, matrix metalloproteinase-9, MT1-matrix metalloproteinase) and their inhibitors (TIMP-1, TIMP-2) in common epithelial tumors of the ovary. Int J Oncol 2000;17:673-681.
12 Kallakury BV, Karikehalli S, Haholu A, et al. Increased expression of matrix metalloproteinases 2 and 9 and tissue inhibitors of metalloproteinases 1 and 2 correlate with poor prognostic variables in renal cell carcinoma. Clin Cancer Res 2001;7:3113-3119.

13 Davidson B, Reich R, Berner A, et al. Ovarian carcinoma cells in serous effusions show altered matrix metalloproteinase-2 and TIMP-2 mRNA levels. European Journal of Cancer 2001;37:2040-2049.

14 Matsuyama Y, Takao S, Aikou T. Comparison of matrix metalloproteinase expression between primary tumors with or without liver metastasis in pancreatic and colorectal carcinomas. J Surg Oncol 2002;80:105-110.

15 Gong YL, Xu GM, Huang WD, et al. Expression of matrix metalloproteinases and the tissue inhibitors of metalloproteinases and their local invasiveness and metastasis in Chinese human pancreatic cancer. J Surg Oncol - Supplement 2000;73:95-99.

16 Kurahara S, Shinohara M, Ikebe T, et al. Expression of matrix metalloproteinaseS, MT-matrix metalloproteinase, and TIMPs in squamous cell carcinoma of the oral cavity: correlations with tumor invasion and metastasis. Head \& Neck 1999;21:627-638.

17 Kawata R, Shimada T, Maruyama S, et al. Enhanced production of matrix metalloproteinase- 2 in human head and neck carcinomas is correlated with lymph node metastasis. Acta Otolaryngol (Stockh) 2002;122: 101-106.

18 Werner JA, Rathcke IO, Mandic R. The role of matrix metalloproteinases in squamous cell carcinomas of the head and neck. Clin Exp Metast 2002;19:275-282.

19 Sutinen M, Kainulainen T, Hurskainen T, et al. Expression of matrix metalloproteinases (matrix metalloproteinase-1 and -2) and their inhibitors (TIMP-1, -2 and -3 ) in oral lichen planus, dysplasia, squamous cell carcinoma and lymph node metastasis. Br J Cancer 1998;77:2239-2245.

20 Yorioka CW, Coletta RD, Alves F, et al. Matrix metalloproteinase-2 and -9 activities correlate with the disease-free survival of oral squamous cell carcinoma patients. Int J Oncol 2002;20:189-194.

21 Tyler LN, Ai L, Zuo C, et al. Analysis of promoter hypermethylation of death-associated protein kinase and p16 tumor suppressor genes in actinic keratoses and squamous cell carcinomas of the skin. Mod Pathol 2003;16:660-664.

22 Katori H, Baba Y, Imagawa Y, et al. Reduction of in vivo tumor growth by MMI-166, a selective matrix metalloproteinase inhibitor, through inhibition of tumor angiogenesis in squamous cell carcinoma cell lines of head and neck. Cancer Lett 2002;178:151-159.

23 Maekawa R, Maki H, Wada T, et al. Anti-metastatic efficacy and safety of MMI-166, a selective matrix metalloproteinase inhibitor. Clin Exp Metast 2000;18: 61-66.

24 Kurokawa H, Yamashita Y, Murata T, et al. Histological grading of malignancy correlates with regional lymph node metastasis and survival of patients with oral squamous cell carcinoma. Fukuoka Igaku Zasshi 1998; 89:225-231.

25 Graflund M, Sorbe B, Hussein A, et al. The prognostic value of histopathologic grading parameters and microvessel density in patients with early squamous cell carcinoma of the uterine cervix. Int J Gynecol Cancer 2002;12:32-41.

26 Adachi Y, Yamamoto H, Itoh F, et al. Clinicopathologic and prognostic significance of matrilysin expression at 
the invasive front in human colorectal cancers. Int J Cancer 2001;95:290-294.

27 Kristensen GB, Abeler VM, Risberg B, et al. Tumor size, depth of invasion, and grading of the invasive tumor front are the main prognostic factors in early squamous cell cervical carcinoma. Gynecol Oncol 1999;74:245-251.

28 Sato T, Iwai M, Sakai T, et al. Enhancement of membrane-type 1-matrix metalloproteinase (MT1-matrix metalloproteinase) production and sequential activation of progelatinase $\mathrm{A}$ on human squamous carcinoma cells co-cultured with human dermal fibroblasts. Br J Cancer 1999;80:1137-1143.

29 Papadopoulou S, Scorilas A, Arnogianaki N, et al. Expression of gelatinase-A (matrix metalloproteinase-
2) in human colon cancer and normal colon mucosa. Tumour Biol 2001;22:383-389.

30 Yano H, Hara A, Murase S, et al. Expression of hepatocyte growth factor and matrix metalloproteinase-2 in human glioma. Brain Tumor Pathol 2001;18: $7-12$.

31 Kanda K, Takahashi M, Murakami Y, et al. The role of the activated form of matrix metalloproteinase-2 in urothelial cancer. BJU Int 2000;86:553-557.

32 Jaalinoja J, Herva R, Korpela M, et al. Matrix metalloproteinase 2 (matrix metalloproteinase-2) immunoreactive protein is associated with poor grade and survival in brain neoplasms. J Neuro-oncol 2000; 46:81-90. 